\title{
Discovery of a new subparsec counterjet in NGC 1275: the inclination angle and the environment
}

\author{
Yutaka Fujita, ${ }^{1 \star}$ and Hiroshi Nagai ${ }^{2}$ \\ ${ }^{1}$ Department of Earth and Space Science, Graduate School of Science, Osaka University, Toyonaka, Osaka 560-0043, Japan \\ ${ }^{2}$ National Astronomical Observatory of Japan, Osawa 2-21-1, Mitaka, Tokyo 181-8588, Japan
}

Accepted XXX. Received YYY; in original form ZZZ

\begin{abstract}
We report the detection of a new feature at the centre of NGC 1275 in the Perseus cluster, hosting the radio source $3 \mathrm{C} 84$. This feature emerges $\sim 2$ mas $(\sim 0.8 \mathrm{pc})$ north of the central core in recent 15 and $43 \mathrm{GHz}$ VLBA images, and seems to be the counterjet to a known radio jet expanding to the south of the core. Apparently, the two jets were born through an outburst around 2005. From the ratio of the apparent lengths of the two jets from the core, we found that the jet angle to the line of sight is $\theta=65^{\circ} \pm 16^{\circ}$, which is not much different from the angle of the outer jets generated by an activity around 1959 and constrains theories on gamma-ray emission from jets. The new northern jet has a strongly inverted spectrum in contrast with the southern jet. This suggests that the central black hole is surrounded by a subparsec-scale accretion disk with the density of $\gtrsim 10^{5} \mathrm{~cm}^{-3}$. The brightness of the counterjet suggests that the disk is highly inhomogeneous. The ambient gas density in the direction of the jet is $\sim 8 \mathrm{~cm}^{-3}$ if the current jet activity is similar to the past average.
\end{abstract}

Key words: galaxies: active - galaxies: individual (3C 84, NGC 1275) - galaxies: jets - radio continuum: galaxies

\section{INTRODUCTION}

NGC 1275 is the central galaxy of the Perseus cluster. It is known as a nearby Seyfert galaxy $(z=0.0176)$ and hosts the compact radio source $3 \mathrm{C} 84$. Cosmic rays accelerated around the central supermassive black hole (SMBH) may be playing an important role in offsetting radiative cooling of the cool core of the cluster (Fujita \& Ohira 2012, 2013). The proximity of the object allows us to make detailed observations about the environment around the SMBH and its activities. Early VLBI observations showed that $3 \mathrm{C} 84$ has complicated structures on a scale of pc; a mushroom-like jet is expanding southward from a bright compact core. The apparent velocity of the southern jet has been estimated to be $\sim 0.3 c$ (Romney et al. 1982; Asada et al. 2006; Lister et al. 2009). From this velocity, it has been suggested that this expanding jet relates to an outburst in 1959 (e.g. Nesterov, Lyuty, \& Valtaoja 1995). The counterjet of this southern jet has been discovered to the north of the core (Vermeulen, Readhead, \& Backer 1994; Walker, Romney, \& Benson 1994). The ratio of the apparent distances of the two jets from the core suggests that the observing angle to the jet direction is $\sim 30^{\circ}-$ $60^{\circ}$ (Walker, Romney, \& Benson 1994; Asada et al. 2006). The angle between the jets and the line of sight has great importance for the gamma-ray spectrum of NGC 1275 (Abdo et al. 2009; Aleksić et al. 2014; Tavecchio \& Ghisellini 2014).

Recently, new activity in the core has been reported. Nagai et

^ E-mail: fujita@vega.ess.sci.osaka-u.ac.jp (YF) al. (2010) showed that a new component (C3 in the paper) emerged in the central subparsec region of the core (C1). They indicated that this component relates to a radio outburst that began in 2005 . Moreover, since the gamma-ray luminosity of NGC 1275 started to increase around 2005 (Dutson et al. 2014), the new component is seemingly associated with the gamma-ray activity. Since the new component is moving toward south from the core, its counterjet, if any, is expected to appear to the north of the core.

In this Letter, we report the discovery of the northern counterjet. From the ratio of the apparent lengths of the southern and northern jets from the core, we estimate the inclination angle of the jets. We also discuss the environment in the vicinity of the SMBH based on the inverted spectrum of the northern jet. We adopt $H_{0}=70 \mathrm{~km} \mathrm{~s}^{-1} \mathrm{Mpc}^{-1}, \Omega_{\mathrm{m}}=0.3$, and $\Lambda=0.7$. For these cosmological parameters, 1 mas corresponds to $0.36 \mathrm{pc}$.

\section{DATA}

We use the calibrated very long base line array (VLBA) data of 3C 84 provided by Monitoring Of Jets in Active galactic nuclei with VLBA Experiments (MOJAVE) ${ }^{1}$ and Boston University (BU) Blazar Monitoring Program ${ }^{2}$. The data were taken on 22 January

\footnotetext{
${ }^{1}$ http://WwW.physics. purdue. edu/MOJAVE/sourcepages/0316+ 413. shtml

2 http://www.bu.edu/blazars/VLBA_GLAST/0316.html
} 


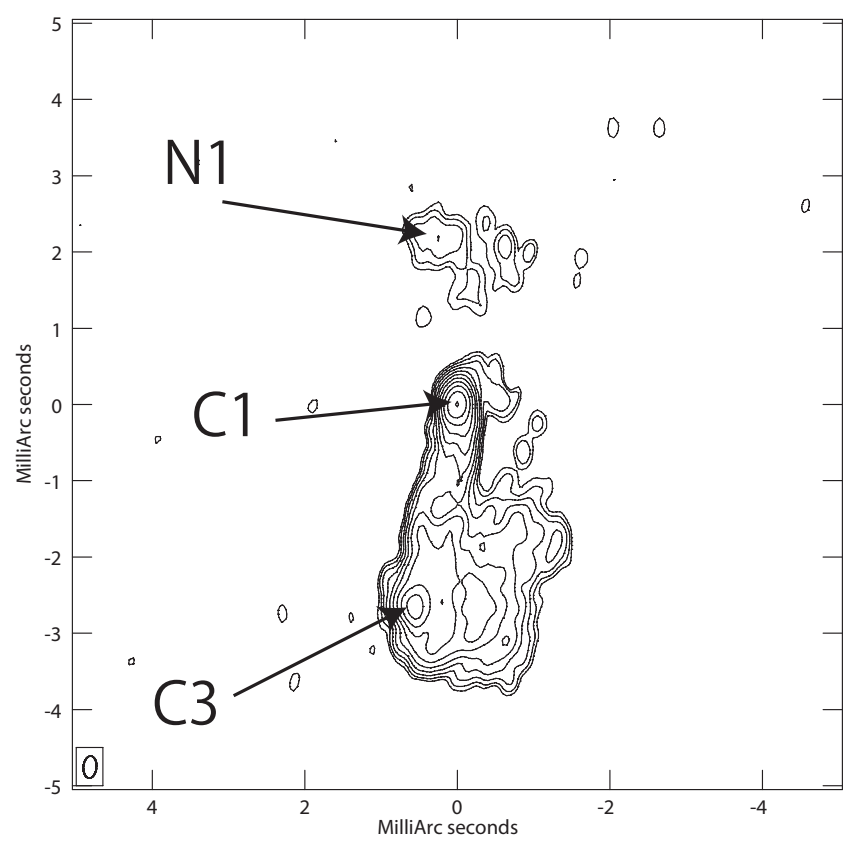

Figure 1. VLBA image of $3 \mathrm{C} 84$ at $43 \mathrm{GHz}$. The core, the southern hotspot and the north component are indicated as $\mathrm{C} 1, \mathrm{C} 3$, and N1, respectively. The contour levels are $2.9 \mathrm{mJy} \times n(n=1,2,4, \ldots . ., 1024)$. Beam FWHM is $0.289 \times 0.171$ mas at a position angle of $-4 .^{\circ} 93$.

2016 at $15 \mathrm{GHz}$ and on 5 December 2015 at $43 \mathrm{GHz}$. The imaging was done using CLEAN algorithm implemented in the Difmap software $^{3}$. Data reduction was performed using the NRAO Astronomical Imaging Processing System (AIPS) ${ }^{4}$.

Fig. 1 shows a $43 \mathrm{GHz}$ image of 3C 84. From the core (C1), a lobe-like feature extends southward, which we call the southern jet. The hotspot $(\mathrm{C} 3)$ is clearly seen in the jet. The hotspot location seems to be shifted to the east compared to the previous $43 \mathrm{GHz}$ VLBA image (Nagai et al. 2016). A new component, which we call $\mathrm{N} 1$, is recognised to the north of the core. While the background noise level is $0.97 \mathrm{mJybeam}^{-1}$, the peak flux of N1 is $23 \mathrm{mJybeam}^{-1}$, which means $24 \sigma$ detection. Fig. 2 shows a $15 \mathrm{GHz}$ image of $3 \mathrm{C} 84$. The north component $\mathrm{N} 1$ can also be seen at the same position in Fig. 1. The background noise level is $1.0 \mathrm{mJy} \mathrm{beam}^{-1}$ and the peak flux of $\mathrm{N} 1$ is $17 \mathrm{mJy}^{-1}$ beam $^{-1}$, which means $17 \sigma$ detection. Thus, it is unlikely that the detection is false.

\section{ANGLE TO THE LINE OF SIGHT}

From the ratio of apparent lengths of the southern and north jets from the core and from the apparent jet velocity, we can estimate the angle of the jets to the line of sight $\theta$. The ratio $D$ is represented by

$$
D=\frac{1+\beta \cos \theta}{1-\beta \cos \theta}
$$

where $\beta$ is the jet velocity normalised by the light speed $c$ (Walker, Romney, \& Benson 1994). The observed velocity of the approach-

\footnotetext{
3 https://science.nrao.edu/facilities/vlba/docs/manuals/ oss2013a/post-processing-software/difmap

4 http://www.aips.nrao.edu/index.shtml
}

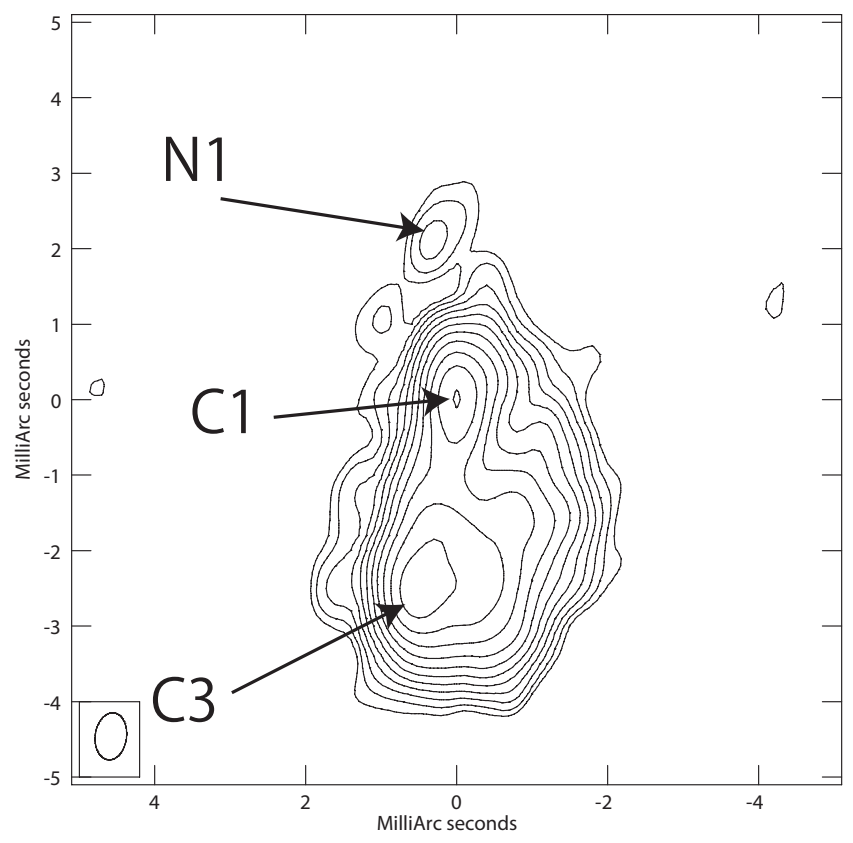

Figure 2. VLBA image of $3 \mathrm{C} 84$ at $15 \mathrm{GHz}$. The core, the southern hotspot and the north component are indicated as $\mathrm{C} 1, \mathrm{C} 3$, and N1, respectively. The contour levels are $3.0 \mathrm{mJy} \times n(n=1,2,4, \ldots . ., 1024)$. Beam FWHM is $0.626 \times 0.416$ mas at a position angle of $-7 .^{\circ} 41$.

ing jet is given by

$$
\beta_{\mathrm{a}}=\frac{\beta \sin \theta}{1-\beta \cos \theta}
$$

(Walker, Romney, \& Benson 1994). Thus, the inclination angle is

$$
\theta=\arctan \frac{2 \beta_{a}}{D-1}
$$

(Asada et al. 2006). Nagai et al. (2010) estimated that the apparent velocity of the approaching component (C3) is $\beta_{\mathrm{a}}=0.23 \pm 0.01$.

We use the $43 \mathrm{GHz}$ image in Fig. 1 to derive the ratio $D$, because the resolution is higher than the $15 \mathrm{GHz}$ image in Fig. 2. We assume that the tips of the southern and northern jets are $\mathrm{C} 3$ and $\mathrm{N} 1$, respectively. The position of $\mathrm{C} 3$ and $\mathrm{N} 1$ are derived by fitting them with a gaussian using AIPS task JMFIT. We ignore the error of the position associated with the fit, because it is much smaller than the beam size $(0.289 \times 0.171 \mathrm{mas})$. The position of the core $(\mathrm{C} 1)$ is derived in the same way, and we assume that it is the position of the black hole. The ratio $D$ is given by the ratio of the distance from the black hole to $\mathrm{C} 3$ to the distance from the black hole to $\mathrm{N} 1$, and it is $D=1.22 \pm 0.16$, where the error comes from the beam size. Thus, we obtain $\theta=65^{\circ} \pm 16^{\circ}$ from equation ( 3 ), and $\beta=0.23 \pm 0.02$ from equations (1) or (2). The obtained angle is not much different from that of the outer pair of 'old' jets $\left(\theta \sim 30^{\circ}-60^{\circ}\right)$ associated with an outburst of 3C 84 around 1959 (Walker, Romney, \& Benson 1994; Asada et al. 2006). Moreover, the jet direction on the sky is similar to that of the old jets (Vermeulen, Readhead, \& Backer 1994; Walker, Romney, \& Benson 1994). These suggest that the jet direction has not much changed over the last half a century. Note that the position of the core may be affected by an opacity-induced core shift. For M 87, the core shift is $0.007-0.01 \mathrm{pc}$ at $43 \mathrm{GHz}$ (Hada et al. 2011), which corresponds to $\sim 0.02$ mas for NGC 1275. Thus, the effect of the core shift is probably negligible. However, Fig. 6 of Walker et al. (2000) shows that the core has a strong northern 
ridge for the inner mas or so at higher frequencies. The position of the peak of the core may depend on resolution due to blending of the inner features. Thus, as an extreme case, we set the position of the black hole at the northern edge of the core $(0.3$ mas north of the peak), and we calculate the jet parameters. We found that $D=1.56 \pm 0.21, \theta=39^{\circ} \pm 10^{\circ}$, and $\beta=0.28 \pm 0.04$. The angle $\theta$ is still fairly large.

We expect that the 'new' jets (C3 and N1) are associated with gamma-ray emission that started to increase around 2005 (Abdo et al. 2009; Dutson et al. 2014). The obtained inclination angle has an implication for theories on gamma-ray radiation from jets in radio galaxies. A popular idea is that NGC 1275 is a misaligned blazar. However, although one-zone synchrotron-self Compton models may explain the observed broadband spectral energy distribution (SED), they require an untypical low bulk-Lorentz factor (Aleksić et al. 2014). Even if two-zone 'spine-layer' jet models are considered, they conflict with the relatively large angle that we obtained $\left(\theta=65^{\circ} \pm 16^{\circ}\right)$. This is because the gamma-rays with energies above a few tens of $\mathrm{GeV}$ are absorbed in the luminous infrared radiation field associated with the fast spine emission (Tavecchio \& Ghisellini 2014). Moreover, in spite of the large inclination angle, the gamma-ray luminosity of NGC 1275 is very large and is comparable to those of blazars (Kataoka et al. 2010). These facts suggest that the misaligned blazar interpretation may not be applied for NGC 1275.

\section{ABSORPTION BY THE ACCRETION DISK}

The spectral index of the radio emission can give us information on absorption toward an object. Thus, we estimate the spectral indices for the southern and the northern components. For that purpose, we make an image at $43 \mathrm{GHz}$ with a convolving beam of $0.626 \times 0.416$ mas to match the resolution of the $15 \mathrm{GHz}$ observation (Fig. 2). Then, we estimate the fluxes of the components $\mathrm{C} 3$ and N1 by fitting them with a gaussian using AIPS task JMFIT for both of the 43 and $15 \mathrm{GHz}$ images. From the ratio of the flux at $43 \mathrm{GHz}$ to that at $15 \mathrm{GHz}$, we find that the spectral index of $\mathrm{C} 3$ is $\alpha_{\mathrm{S}}=-0.91$ and that of $\mathrm{N} 1$ is $\alpha_{\mathrm{N}}=1.61$, where the spectral index is defined as $S \propto v^{\alpha}$. The index of C3 is the one for a typical synchrotron emission region, which means that the component is not much affected by absorption. On the other hand, N1 has an inverted spectrum and it is likely to be absorbed. Considering the inclination angle of the jets $\left(\theta \sim 65^{\circ}\right)$, a natural explanation is that the black hole is surrounded by an optically-thick accretion disk, which is perpendicular to the jets and obscures only the northern component.

If the southern and the northern components are symmetric, and if there is no absorption, the ratio of brightness between the two components (C3 and $\mathrm{N} 1)$ is written as

$$
R=\left(\frac{1+\beta \cos \theta}{1-\beta \cos \theta}\right)^{\alpha+m},
$$

where $m$ is ether 2 or 3 for a continuous jet or single component, respectively (Walker, Romney, \& Benson 1994). Using the values of $\theta$ and $\beta$ derived in section 3 and assuming that $\alpha=\alpha_{S}$, we can predict that the brightness ratio should be $R=1.8 \pm 0.6$ for $m=2$, and $R=2.1 \pm 1.0$ for $m=3$. However, the observed ratios are much larger and they are $R_{\mathrm{obs}}=45$ at $43 \mathrm{GHz}$ and $R_{\mathrm{obs}}=$ 600 at $15 \mathrm{GHz}$. These large values clearly indicate that the north component is actually absorbed by the accretion disk.

The most likely explanation for the inverted spectrum and the large ratio of brightness is free-free absorption (Levinson, Laor, \& Vermeulen 1995). The free-free optical depth can be written as

$$
\tau_{\mathrm{ff}} \approx 5.6 \times 10^{-8} \bar{g}\left(\frac{T}{10^{4} \mathrm{~K}}\right)^{-3 / 2}\left(\frac{n_{\mathrm{e}}}{\mathrm{cm}^{-3}}\right)^{2}\left(\frac{v}{\mathrm{GHz}}\right)^{-2}\left(\frac{L}{\mathrm{pc}}\right),
$$

where $T$ is the temperature of the absorbing medium, $n_{\mathrm{e}}$ is the electron density (e.g. Rybicki \& Lightman 1979), and $L$ is the disk depth along the line of sight. Here, we assumed that the absorbing medium is a pure hydrogen plasma with a uniform density, and that the electron density equals the proton density. For $T \sim 10^{4} \mathrm{~K}$ and $v \sim 15-43 \mathrm{GHz}$, the Gaunt factor is $\bar{g} \sim 4$ (e.g. Hummer 1988). We can estimate the optical depth from the relation of

$$
R_{\mathrm{obs}}=R \exp \left(\tau_{\mathrm{ff}}\right) .
$$

For $m=2$, the optical depth is $\tau_{\mathrm{ff}} \approx 3.2$ at $43 \mathrm{GHz}$, and $\tau_{\mathrm{ff}} \approx 5.8$ at $15 \mathrm{GHz}$. We can calculate the density of the disk using equation (5). Assuming that $T=10^{4} \mathrm{~K}$ and $L=0.8 \mathrm{pc}$, which is the apparent distance between $\mathrm{C} 1$ and $\mathrm{N} 1$, the density is $n_{\mathrm{e}} \approx 1.9 \times 10^{5} \mathrm{~cm}^{-3}$ for $43 \mathrm{GHz}$, and $n_{\mathrm{e}} \approx 8.9 \times 10^{4} \mathrm{~cm}^{-3}$ for $15 \mathrm{GHz}$. The density is not much different when $m=3$. If the disk is thinner or $L$ is smaller, the density is higher. Thus, we conclude that the gas density is $n_{\mathrm{e}} \gtrsim$ $10^{5} \mathrm{~cm}^{-3}$.

It is interesting to note that the ratio of the observed optical depths at 43 and $15 \mathrm{GHz}$ suggests that $\tau_{\mathrm{ff}} \propto v^{-0.6}$, which is different from $\tau_{\mathrm{ff}} \propto v^{-2}$ in equation (5). One explanation may be that the absorbing medium is highly inhomogeneous and it consists of regions of $\tau_{\mathrm{ff}} \gg 1$ and $\tau_{\mathrm{ff}} \ll 1$. If this is the case, the averaged optical depth is less dependent on the observational frequency.

If a temperature of $T=10^{4} \mathrm{~K}$ is assumed, the optical depth $\tau_{\mathrm{ff}}$ we obtained and equation (5) mean that the emission measure $(\mathrm{EM})$ is $n_{\mathrm{e}}^{2} L \sim 2.7 \times 10^{10} \mathrm{pc} \mathrm{cm}^{-6}$ for $43 \mathrm{GHz}$ and $\sim 6.2 \times 10^{9} \mathrm{pc} \mathrm{cm}^{-6}$ for $15 \mathrm{GHz}$ at the position of $\mathrm{N} 1$ (a projected distance of $d=0.8 \mathrm{pc}$ from the core). On the other hand, Walker et al. (2000) found that the EM at $d \sim 1.4-3.6 \mathrm{pc}$ is $n_{\mathrm{e}}^{2} L \sim 5.7 \times 10^{8}(d / 2.7 \mathrm{pc})^{-1.5} \mathrm{pccm}^{-6}$ based on the data taken in January 1995 or $n_{\mathrm{e}}^{2} L \sim 4.8 \times 10^{8}(d / 2.7 \mathrm{pc})^{-1.8} \mathrm{pccm}^{-6}$ based on the data taken in October $1995^{5}$. If we extrapolate these to $d=0.8 \mathrm{pc}$, the EM is $n_{\mathrm{e}}^{2} L \sim 4 \times 10^{9} \mathrm{pc} \mathrm{cm}^{-6}$. Thus, the EM we obtained is $\sim 1.5-7$ times larger than the extrapolated one. However, this may be because Walker et al. (2000) derived their profiles for a fairly narrow range of distance. For $d \sim 3-30 \mathrm{pc}$, Silver, Taylor, \& Vermeulen (1998) indicated that the EM is $n_{\mathrm{e}}^{2} L \sim 1 \times 10^{6}(d / 30 \mathrm{pc})^{-2.6} \mathrm{pc} \mathrm{cm}^{-6}$ if $T=10^{4} \mathrm{~K}$. If we fit the above data over the whole range $(d \sim 0.8-30 \mathrm{pc})$ with a single power-law, they are well represented by $n_{\mathrm{e}}^{2} L \sim 7 \times 10^{9}(d / \mathrm{pc})^{-2.6} \mathrm{pc} \mathrm{cm}^{-6}$.

\section{GAS DENSITY IN THE JET DIRECTION}

Fujita et al. (2016) discussed the density of the surrounding medium in the direction of the outer old jets or perpendicular to the accretion disk in NGC 1275. Here, we apply this argument to the new jets shown in Figs. 1 and 2.

The momentum balance along the jet is given by

$$
L_{\mathrm{j}} / c=\rho_{\mathrm{h}} V_{\mathrm{h}}^{2} A_{\mathrm{h}},
$$

where $L_{\mathrm{j}}$ is the kinematic power of the jet, $\rho_{\mathrm{h}}$ is the density of the ambient gas just ahead of the jet, $V_{\mathrm{h}}$ is the velocity the hotspot

5 Walker et al. (2000) adopted $H_{0}=75 \mathrm{kms}^{-1} \mathrm{Mpc}^{-1}$ and we have corrected the difference from ours. 
(the jet head), and $A_{\mathrm{h}}$ is the cross-section area of the jet head. The density profile of the ambient gas is assumed to be

$$
\rho(r)=\rho_{\mathrm{h}}\left(r / r_{\mathrm{h}}\right)^{-X},
$$

where $r$ is the three-dimensional distance from the black hole and $r_{\mathrm{h}}$ is the position of the jet head. Note that since the new jets are propagating in the old jets, the ambient gas here means the gas in the old jets. We implicitly assume that the old jets are filled with a significant fraction of thermal gas. The age of the jet is given by

$$
t_{\text {age }}=\frac{2 r_{\mathrm{h}}}{4-X}\left(\frac{L_{\mathrm{j}}}{\rho_{\mathrm{h}} c A_{\mathrm{h}}}\right)^{-1 / 2}
$$

from equation (8) in Fujita et al. (2016) ${ }^{6}$.

The apparent distance between the black hole and C3 is 2.7 mas. Considering the inclination angle $\left(\theta \sim 65^{\circ}\right)$, the actual jet length is $r_{\mathrm{h}} \sim 1.1 \mathrm{pc}$. The width of the southern lobe at the position of $\mathrm{C} 3$ is $\sim 1.9$ mas (Fig 1), and thus the area of the jet head is $A_{\mathrm{h}} \sim 0.36 \mathrm{pc}^{2}$. The jet velocity is $V_{\mathrm{h}} \sim 0.23 c$ (section 3 ). Following Fujita et al. (2016), we assume $X=1.5$, although results are insensitive to the value of $X$. We also assume that the current jet power $L_{\mathrm{j}}$ is comparable to the average jet power in the past $\left(\sim 10^{7} \mathrm{yr}\right)$. From the size of X-ray cavities, Rafferty et al. (2006) estimated that the average power is $P_{\text {cav }} \sim 1.5 \times 10^{44} \mathrm{erg} \mathrm{s}^{-1}$.

Based on these assumptions, we can derive $\rho_{\mathrm{h}}$ so that the condition of $2 L_{\mathrm{j}}=P_{\text {cav }}$ is satisfied using equation (7). We find that the number density of the ambient gas at $r=r_{\mathrm{h}}$ is $n_{\mathrm{e}}\left(r_{\mathrm{h}}\right) \sim 8.1 \mathrm{~cm}^{-3}$. The age of the jets is $t_{\mathrm{age}}=12 \mathrm{yr}$ (equation (9)), which means that the jets was born in 2003 or 2004 . The birth time is not much different from the beginning of the recent activity around 2005 (Abdo et al. 2009; Dutson et al. 2014). Note that for the outer old jets, Fujita et al. (2016) obtained $n_{\mathrm{e}}\left(r_{\mathrm{h}}\right) \sim 0.096 \mathrm{~cm}^{-3}$ for $2 L_{\mathrm{j}}=0.37 P_{\text {cav }}$ and $r_{\mathrm{h}}=5.6$ pc. If $2 L_{\mathrm{j}}=P_{\text {cav }}$, the density is $n_{\mathrm{e}}\left(r_{\mathrm{h}}\right) \sim 0.26 \mathrm{~cm}^{-3}$.

\section{TIME VARIABILITY}

It is interesting to note that there was a feature at the position of $\mathrm{N} 1$ in the full resolution 15 and $22 \mathrm{GHz}$ images shown in Fig. 5 of Walker et al. (2000) and in the deep $23 \mathrm{GHz}$ image shown in Fig. 1 of Walker \& Anantharamaiah (2003). The observations were made in October 1995 for the former and in September 1998 for the latter. Although $15 \mathrm{GHz}$ data (MOJAVE) have been available since 1999, noticeable features are not recognised at the position of $\mathrm{N} 1$ before September 2015. For $43 \mathrm{GHz}$ data (BU), which have been available since 2010, a noticeable feature appears at the position of N1 in December 2015. Since both MOJAVE and BU observations are not deep, we cannot deny that dim features had been overlooked.

If there has always been a feature there, it might be the result of some other effect. For example, there may be a groove on the accretion disk, and when a bright component in the jet (e.g. a knot) passes behind the groove, $\mathrm{N} 1$ may be brightened. If the emergence of N1 at the end of 2015 is attributed to the passage of the hot spot of the new jet behind the groove, the main conclusions of this paper do not change. On the other hand, N1 may be associated with an internal structure in the jet that stays at the same position (e.g. a recollimation shock) while the luminosity changes. Long and deep observations are required to investigate these possibilities.

\footnotetext{
${ }^{6}$ In Fujita et al. (2016), the density profile is given by $\rho(r)=\rho_{\mathrm{B}}\left(r / r_{\mathrm{B}}\right)^{-X}$, where $r_{\mathrm{B}}$ is the Bondi radius and $\rho_{\mathrm{B}}=\rho\left(r_{\mathrm{B}}\right)$. Thus, equation (9) in this letter can be obtained using the relation of $\rho_{\mathrm{h}} r_{\mathrm{h}}^{X}=\rho_{\mathrm{B}} r_{\mathrm{B}}^{X}$.
}

\section{CONCLUSIONS}

We discovered a new feature $\sim 2$ mas $(\sim 0.8 \mathrm{pc})$ north of the central core of NGC 1275 (3C 84) at 15 and $43 \mathrm{GHz}$ with VLBA. This feature is considered to be the counterjet of the jet expanding southward from the core that launched around 2005. From the ratio of the lengths of the two jets, we estimated the inclination angle of the jet and found that it is $\theta=65^{\circ} \pm 16^{\circ}$, which is not much different from that of the outer old jets that launched around 1959. The northern jet has a strongly inverted spectrum, which indicates that it is absorbed by an accretion disk around the SMBH. From the brightness of the northern jet, we calculated the density of the disk and found that it is $\gtrsim 10^{5} \mathrm{~cm}^{-3}$. We also indicated that the disk may be highly inhomogeneous. Assuming that the current jet power is not much different from the past average, we derived the ambient gas density in the jet direction $\left(\sim 8 \mathrm{~cm}^{-3}\right)$.

\section{ACKNOWLEDGEMENTS}

We would like to thank the referee for useful comments. We are grateful to Motoki Kino, and Kiyoaki Wajima for helpful discussion. This work was supported by MEXT KAKENHI No. 15K05080 (YF). HN is supported by MEXT KAKENHI No. 15K17619. This research has made use of data from the MOJAVE database that is maintained by the MOJAVE team (Lister et al. 2009). This study makes use of $43 \mathrm{GHz}$ VLBA data from the VLBA-BU Blazar Monitoring Program (VLBA-BU-BLAZAR; http://www.bu.edu/blazars/VLBAproject.html), funded by NASA through the Fermi Guest Investigator Program. The VLBA is an instrument of the National Radio Astronomy Observatory. The National Radio Astronomy Observatory is a facility of the National Science Foundation operated by Associated Universities, Inc.

\section{REFERENCES}

Abdo A. A., et al., 2009, ApJ, 699, 31

Aleksić J., et al., 2014, A\&A, 564, A5

Asada K., Kameno S., Shen Z.-Q., Horiuchi S., Gabuzda D. C., Inoue M., 2006, PASJ, 58, 261

Dutson K. L., Edge A. C., Hinton J. A., Hogan M. T., Gurwell M. A., Alston W. N., 2014, MNRAS, 442, 2048

Fujita Y., Kawakatu N., Shlosman I., Ito H., 2016, MNRAS, 455, 2289

Fujita Y., Ohira Y., 2012, ApJ, 746, 53

Fujita Y., Ohira Y., 2013, MNRAS, 428, 599

Hada K., Doi A., Kino M., Nagai H., Hagiwara Y., Kawaguchi N., 2011, Natur, 477, 185

Hummer D. G., 1988, ApJ, 327, 477

Kataoka J., et al., 2010, ApJ, 715, 554

Levinson A., Laor A., Vermeulen R. C., 1995, ApJ, 448, 589

Lister M. L., et al., 2009, AJ, 138, 1874-1892

Lister M. L., et al., 2009, AJ, 137, 3718-3729

Nagai H., et al., 2010, PASJ, 62, L11

Nagai H., Chida H., Kino M., Orienti M., D’Ammando F., Giovannini G., Hiura K., 2016, AN, 337, 69

Nesterov N. S., Lyuty V. M., Valtaoja E., 1995, A\&A, 296, 628

Romney J., Alef W., Pauliny-Toth I., Preuss E., Kellermann K., 1982, IAUS, 97, 291

Rafferty D. A., McNamara B. R., Nulsen P. E. J., Wise M. W., 2006, ApJ, 652,216

Rybicki G. B., Lightman A. P., 1979 (Wiley, New York)

Silver C. S., Taylor G. B., Vermeulen R. C., 1998, ApJ, 502, 229

Tavecchio F., Ghisellini G., 2014, MNRAS, 443, 1224

Vermeulen R. C., Readhead A. C. S., Backer D. C., 1994, ApJ, 430, L41 
Walker R. C., Anantharamaiah K. R., 2003, AJ, 125, 1756

Walker R. C., Dhawan V., Romney J. D., Kellermann K. I., Vermeulen R. C., 2000, ApJ, 530, 233

Walker R. C., Romney J. D., Benson J. M., 1994, ApJ, 430, L45

This paper has been typeset from a $\mathrm{T}_{\mathrm{E}} \mathrm{X} / \mathrm{L} \mathrm{ET} \mathrm{E}$ file prepared by the author. 